\title{
ON COMPARING DIFFERENT TESTS \\ "1 \\ OF THE SAME HYPOTHESIS
}

\author{
by \\ Carmen Adela Pérez \\ 川1
}

Thesis submitted to the Graduate Faculty of the Virginia Polytechnic Institute

in candidacy for the degree of

MASTER OF SCIENCE

in

Statistics

October 1959

Blacksburg, Virginia 
TABLE OF CONTENTS

CHAPTER

PAGE

I

IINTRODUCTION AND SUMMARY. •....... 3

II

TESTS OF LOCATION . . . . . . . . 5

2.1 Generalizations. ....... 10

III TESTS OF DISPERSION . . . . . . . . 13

IV THE SIGN TEST AND THE T-TEST. . . . . . $2 I$

ACKMONLEDGRENTS •. •. . . . . . 30

REFERTICES. . . . . . . . . $3 I$

VITA. . . . . . . . . . . 32 


\section{INTRODUCTION AND SUMMARY}

The usual procedure for comparing tests of the same hypothesis is based on their power functions. This is done by plotting the power curves of each test for a fixed Type I error. The power curve of the best test (î́ such a test exists) will lie above the curve of any other test with the same level of significance.

Suppose we have the standard test of a null hypothesis $H_{0}$ based on the statistic $u_{I}$. Sometimes it is convenient to use a quicker, although less powerful, test based on a statistic $u_{2}$. We want to determine the degree of agreement between these two tests. The method of power curves, however, only gives an indication of the long-run behavior of each test and not of the extent to which their results actually agree when applied to the same problem.

In this thesis two nethods for comparing standard and quick tests are proposed. The first method consists of determining the probability of establishing significance with $u_{2}$ at a level $\beta$, given that $u_{I}$ is just significant at level $\alpha$. The second approach determines how significant the expected value of $u_{2}$ is, given that $u_{1}$ is just significant at level $\alpha_{\circ}$

We apply these two alternative procedures to compare:

(a) tests of location in a sample from a normal population with known variance; 
(b) tests of dispersion, namely those based on the standard deviation, mean deviation, and range in a normal sample with unknown mean;

(c) the sign test and the paired t-test, again in a sample from a normal population.

The subject of this thesis and some further issues have also been treated in a slightly more advanced fashion in a Report by David and Perez (1959). 


\section{TESTS OF LOCATION}

Let us consider a random sample $x_{1}, x_{2}, \ldots, x_{n}$ taken from a normal population with variance equal to 1. It is desired to test the null hypothesis $H_{0}: \mu=0$ against the alternative $\mathrm{H}_{I}: \mu>0$. The standard procedure in this case is to use the statistic $u_{1}=\bar{x} \sqrt{ }$, which is a unit normal variable, and for an a level of significance reject $H_{0}$ when $u_{I}>u_{a}$. This is a uniformly most powerful test of $\mathrm{H}_{\mathrm{O}^{\circ}}$

Restricting ourselves to the first $k$ observations only, a quicker test would consist of rejecting $\mathrm{H}_{0}$ when $u_{2}>u_{\alpha}$, where $u_{2}=\bar{x}_{k} \sqrt{ } k$. We shall now apply two methods to compare the statistics $u_{1}$ and $u_{2}$ of the efficient and quick tests respectively.

(a) To determine the probability that $v_{2}$ exceeds its $\beta$ significance point given that $u_{1}$ is just significant at level $a$. That is, to rind

$$
P=\operatorname{Pr}\left(u_{2}>u_{\beta} \mid u_{1}=u_{\alpha}\right) \text {. }
$$

From the nornal density function of two variables $x$ and $y$, with means $\mu_{x}, \mu_{y}$, variances $\sigma_{x}^{2}, \sigma_{y}^{2}$, and correlation coefficient $\rho$, the conditional density $f(x \mid y)$ is found to be a univariate normal with mean $\mu_{x}+\rho \frac{\sigma_{x}}{\sigma_{y}}\left(y-\mu_{y}\right)$ and variance $\sigma_{x}^{2}\left(1-\rho^{2}\right)$. Thus, under $H_{0}, f\left(u_{2} \mid u_{1}^{y}=u_{\alpha}\right)$ is $\mathbb{N}\left(\rho u_{\alpha}, 1-\rho^{2}\right)$ and therefore we have

$$
P=\operatorname{Pr}\left[u>\frac{u_{\beta}-\rho u_{\alpha}}{\sqrt{ }\left(1-\rho^{2}\right)}\right]
$$


The correlation coefficient $\rho$ is, by definition,

$$
\rho\left(u_{1}, u_{2}\right)=\frac{\operatorname{cov}\left(u_{1}, u_{2}\right)}{\sqrt{\operatorname{var} u_{1} \operatorname{var} u_{2}}}=\operatorname{cov}\left(u_{1}, u_{2}\right) \text { 。 }
$$

and

$$
\begin{aligned}
\operatorname{cov}\left(u_{I}, u_{2}\right) & =\sqrt{n k} \operatorname{cov}\left(\bar{x}, \bar{x}_{k}\right) \\
& =\frac{1}{\sqrt{n k}} \operatorname{cov}\left[\sum_{i=1}^{n} x_{i}, \sum_{i=1}^{k} x_{i}\right] \\
& =\frac{1}{\sqrt{n k}} \sum_{i, j=1}^{k} \operatorname{cov}\left(x_{i}, x_{j}\right) \vdots
\end{aligned}
$$

but $\operatorname{cov}\left(x_{i}, x_{j}\right)=\operatorname{var}\left(x_{i}\right)=I,(i=j)$, and since the observations are independent, $\operatorname{cov}\left(x_{i}, x_{j}\right)=0,(i \neq j)$. Therefore,

$$
p=\operatorname{cov}\left(u_{1}, u_{2}\right)=\frac{k}{\sqrt{n k}}=/ \frac{k}{n} \text {. }
$$

From equation ( $(1)$, it $c a n$ be seen that $P \leq \frac{1}{z}$ when $\alpha=\beta$. Hence it seems appropriate to choose $\beta>\alpha \quad\left(u_{\beta}<u_{\alpha}\right)$.

In Table 1 we have the values of $P$ obtained for $\alpha=0.01$, $\beta=0.05$ and for values of $\rho \geq 0.5$.

\section{Table 1}

\begin{tabular}{|l|l|l|l|l|l|l|l|l|}
\hline$\rho$ & 0.5 & 0.6 & 0.7 & 0.3 & 0.9 & 0.95 & 0.98 & 1 \\
\hline$P$ & .289 & .378 & .491 & .641 & .848 & .965 & .999 & 1 \\
\hline
\end{tabular}

The approach of $\mathrm{P}$ to unity is better illustrated in Fig. I, where $P$ is plotted as $a$ function of $\rho$. 
Fig. 1. $P=\operatorname{Pr}\left(u_{2}>1.645 / u_{1}=2.326\right)$ as a function of $p$, the correlation coefficient of the unit normal variates $u_{1}, u_{2}$.

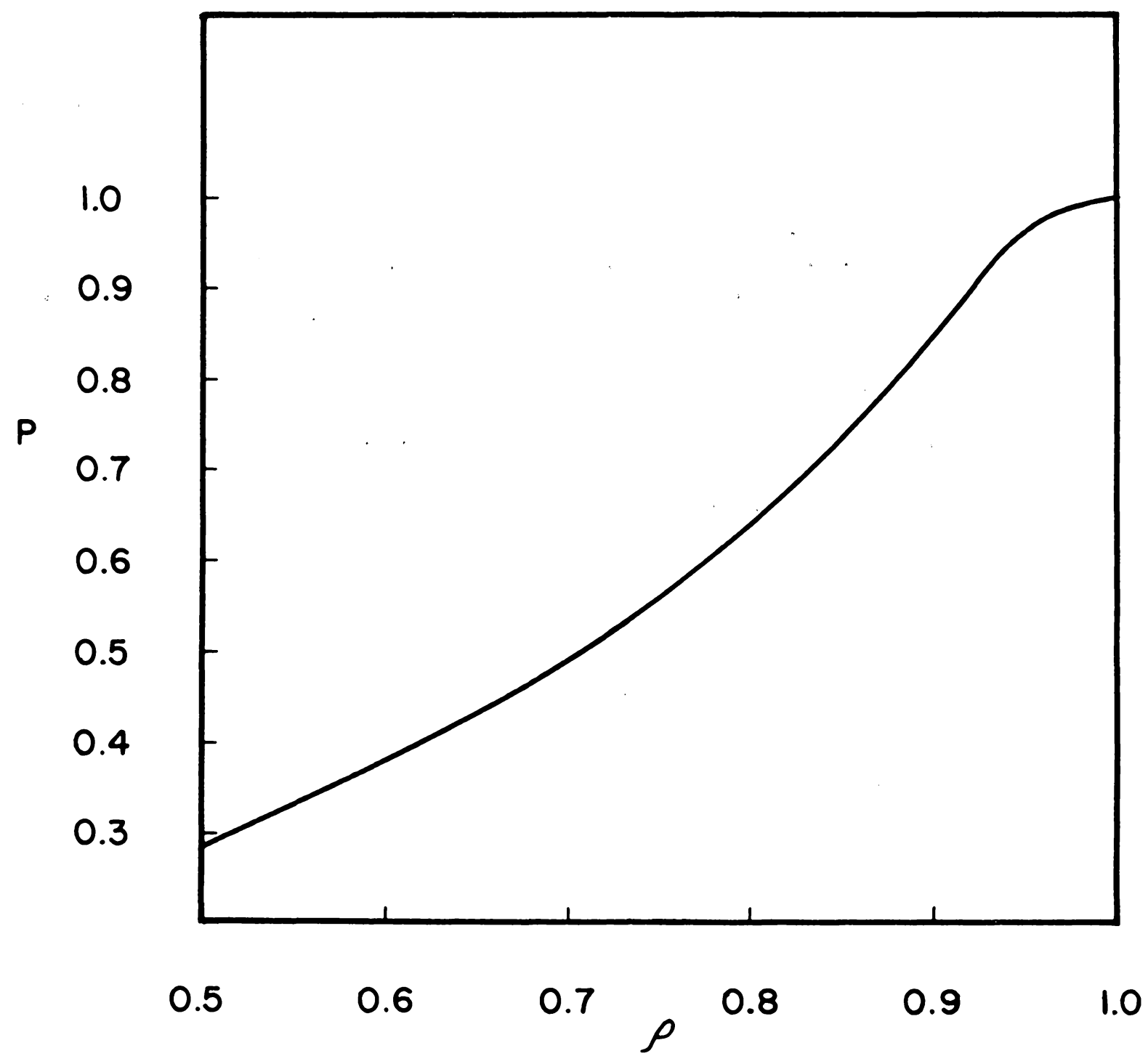


(b) The second approach, which is generally simpler, is to determine the significance level corresponding to the expected value of $u_{2}$ given that $u_{1}$ is just significant at a level $\alpha$. This conditional expectation is given by

$$
\mathcal{C}\left(u_{2} \mid u_{1}=u_{\alpha}\right)=\rho u_{\alpha} \text {. }
$$

The corresponding level of significance or "equivalent Type I error" of the quick test is then

$$
\gamma=\operatorname{Pr}\left(u_{2}>\rho u_{\alpha}\right) \text {. }
$$

Table 2 gives the values of $\gamma$ for $\rho \geq 0.5$ and $\alpha=0.01,0.05$.

Table 2

\begin{tabular}{|l|c|c|}
\hline$\rho$ & $\begin{array}{c}a=0.01 \\
\gamma\end{array}$ & $\begin{array}{c}\alpha=0.05 \\
\gamma\end{array}$ \\
\hline 0.5 & .1224 & .2054 \\
0.6 & .0814 & .1618 \\
0.7 & .0517 & .1248 \\
0.3 & .0314 & .0941 \\
0.9 & .0181 & .0694 \\
0.95 & .0136 & .0591 \\
0.98 & .0113 & .0535 \\
1.00 & .0100 & .0500 \\
\hline
\end{tabular}

In Fig. $2 \gamma$ is plotted as a function of $\rho$ for both values of $\alpha$ 。 
Fig. 2. $r=\operatorname{Pr}\left(u_{2}>\rho u_{\alpha}\right)$

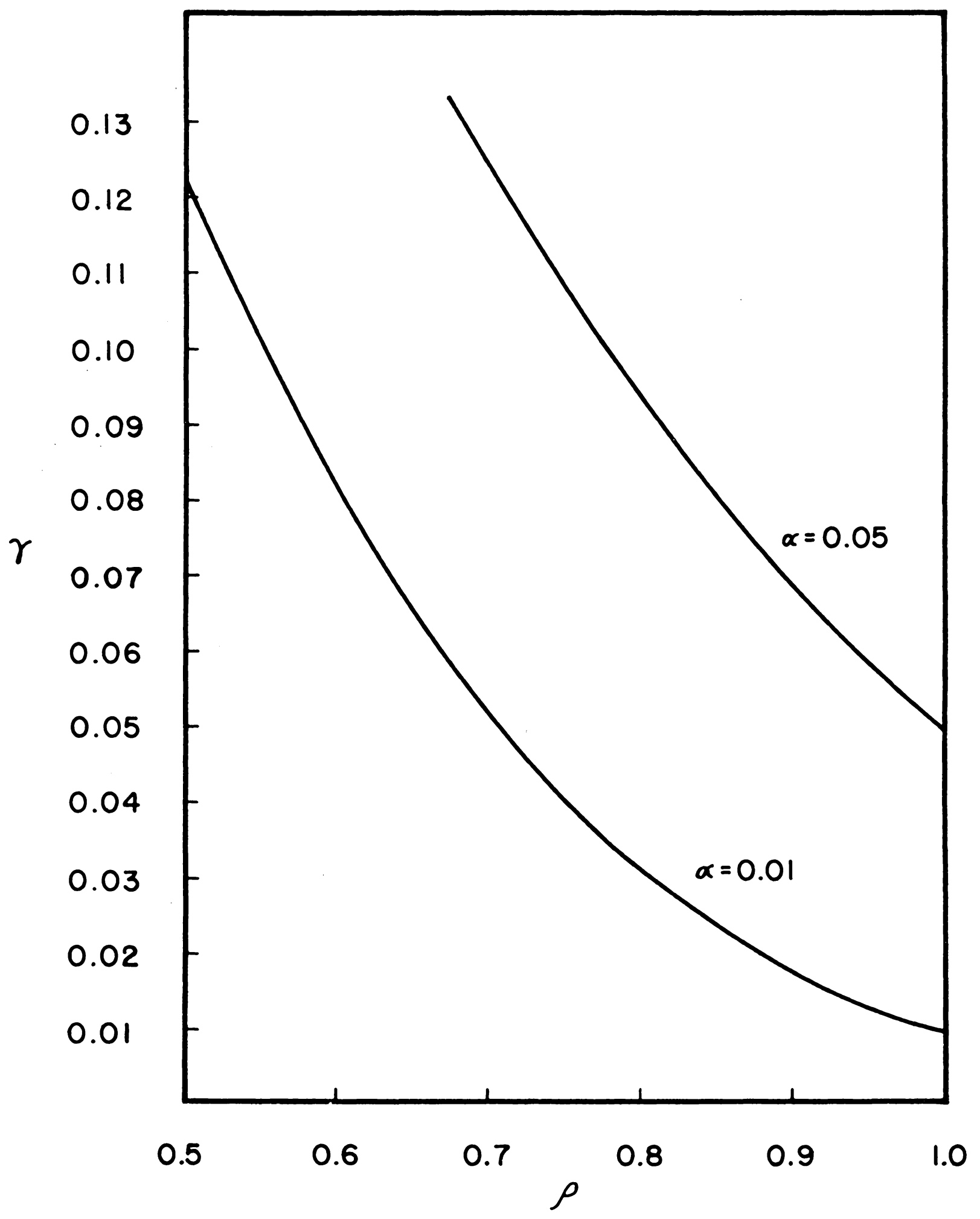




\subsection{Generalizations}

The above procedure can be applied to the more general case of comparing $\bar{x}$ with any linear unbiased estinator of $\mu$ (e.g. median, mid-range) which we will tern $\underline{m}$.

We will first determine $P$ which can be expressed as

$$
\begin{array}{rlr}
P & =\operatorname{Pr}\left(m>m_{\beta} \mid \bar{x}=\bar{x}_{\alpha}\right) \quad\left(\bar{x}_{\alpha}=\lambda_{\alpha} / \sqrt{ } n\right) \\
& =\operatorname{Pr}\left(m-\bar{x}>m_{\beta}-\bar{x} \mid \bar{x}=\bar{x}_{\alpha}\right) &
\end{array}
$$

Now $m-\bar{x}$ can be shown to be independent of $\bar{x}$. We have

$$
\begin{aligned}
f\left(x_{1}, x_{2}, \ldots, x_{n}\right) & =\frac{1}{(2 \pi)^{n / 2}} e^{-\frac{2}{2} \Sigma\left(x_{i}-\mu\right)^{2}} \\
& =\frac{1}{(2 \pi)^{n / 2}} e^{-\frac{1}{2} \Sigma\left(x_{i}-\bar{x}\right)^{2}} e^{-\frac{1}{2} n(\bar{x}-\mu)^{2}}
\end{aligned}
$$

Applying Helmert's orthogonal transformation to the $x^{\text {'s }}$ we get

$$
\begin{aligned}
& x_{1}=\frac{y_{1}}{\sqrt{n}}+\frac{y_{2}}{\sqrt{1.2}}+\frac{y_{3}}{\sqrt{2.3}}+\ldots+\frac{y_{y_{1}}}{\sqrt{(n-1) n}} \\
& \mathrm{x}_{2}=\frac{\mathrm{y}_{1}}{\sqrt{\mathrm{n}}}-\frac{\mathrm{y}_{2}}{\sqrt{1.2}}+\frac{\mathrm{y}_{3}}{\sqrt{2 \cdot 3}}+\ldots+\frac{\mathrm{y}_{\mathrm{n}}}{\sqrt{(\mathrm{n}-1)_{\mathrm{n}}}} \\
& x_{3}=\frac{y_{1}}{\sqrt{n}} \quad-\frac{2 y_{3}}{\sqrt{2.3}}+\ldots+\frac{y_{n}}{\sqrt{(n-1) n}} \\
& x_{n}=\frac{y_{1}}{\sqrt{n}} \quad-\frac{(n-1) y_{n}}{\sqrt{(n-1) n}}
\end{aligned}
$$


where $y_{I}=\sqrt{ } \bar{x}$ and $\Sigma\left(x_{i}-\bar{x}\right)^{2}$ is formed by

$$
\begin{aligned}
& x_{1}-\frac{y_{1}}{\sqrt{n}}=\frac{y_{2}}{\sqrt{1.2}}+\frac{y_{3}}{\sqrt{2.3}}+\ldots+\frac{y_{n}}{\sqrt{(n-1)_{n}}} \\
& x_{2}-\frac{y_{1}}{\sqrt{n}}=-\frac{y_{2}}{\sqrt{1.2}}+\frac{y_{3}}{\sqrt{2.3}}+\ldots+\frac{y_{n}}{\sqrt{(n-1)_{n}}} \\
& x_{3}-\frac{y_{1}}{\sqrt{n}}=-\frac{2 y_{3}}{\sqrt{2.3}}+\ldots+\frac{y_{n}}{\sqrt{(n-1)_{n}}} \\
& \vdots y_{n}-\frac{y_{1}}{\sqrt{n}}=
\end{aligned}
$$

Therefore $\Sigma\left(x_{i}-\bar{x}\right)^{2}=y_{2}^{2}+y_{3}^{2}+\ldots y_{n}^{2}$ is a function of the $y_{j}(j=2, \ldots, n)$. Now (I) can be written as

$$
\begin{aligned}
f\left(y_{1}, y_{2}, \ldots, y_{n}\right) & =\frac{1}{(2 \pi)^{n / 2}} e^{-\frac{1}{2} \sum_{2}^{n} y_{j}^{2}} e^{-\frac{1}{2} n\left(y_{1}-\mu \sqrt{ }\right)^{2}} \\
& =f\left(y_{2}, y_{3}, \ldots, y_{n}\right) f\left(y_{1}\right) .
\end{aligned}
$$

Therefore $\mathrm{y}_{I}$ is independent of the joint distribution of $\mathrm{y}_{2}, \mathrm{y}_{3}, \ldots, \mathrm{y}_{\mathrm{n}}$ and hence of any function of $\mathrm{y}_{2}, \ldots, \mathrm{y}_{\mathrm{n}}$. Since $m-\bar{x}$ can be expressed as

$$
m-\bar{x}=\frac{1}{n}\left(m-x_{1}+m-x_{2}+\ldots+m-x_{n}\right)
$$

which is a linear function of the differences of the observations and hence of the $y_{j}$, it follows that $n-\bar{x}$ is also independent or $\mathrm{y}_{1}=\overline{\mathrm{x}} \sqrt{ } \mathrm{n}$. Hence

$$
P=\operatorname{Pr}\left(m-\bar{x}>m_{\beta}-\bar{x}_{\alpha}\right) \text {. }
$$


The second approach is isually easier to apply. Thus, the conditional expectation of in given that $\bar{x}$ is just significant at level $a$ is given by

$$
\begin{aligned}
\mathfrak{C}\left(m \mid \bar{x}=\bar{x}_{\alpha}\right) & =\mathfrak{C}\left(m-\bar{x}+\bar{x} \mid \bar{x}=\bar{x}_{\alpha}\right) \\
& =\mathfrak{E}(m-\bar{x})+\bar{x}_{\alpha} \\
& =\bar{x}_{C i} .
\end{aligned}
$$

Therefore the "equivalent Type I error" of $\mathrm{m}$ is

$$
r=\operatorname{Pr}\left(m>\bar{x}_{\alpha}\right) \text {. }
$$

It should be noted that since we are considering conditional probabilities and expectations given the statistic $\overline{\mathrm{x}}$ which is sufficient for $\mu, \mathrm{P}$ and $\gamma$ do not depend on $\mu$, that is, hold on $\mathrm{H}_{1}$ a.s well as on $\mathrm{H}_{\mathrm{O}}$. 
III. TESTS OF DISPERSTON

Again, let us consider a randorn sample $x_{1}, x_{2}, \ldots, x_{n}$ from a normal population with mean unknown. We wish to test the composite null hypothesis $\mathrm{H}_{0}: 0=1$ against the alternative $\mathrm{H}_{I}: \sigma>I$.

We will consider three estimators of $\sigma$, namely,

i) The standard deviation $s=\sqrt{\frac{\Sigma\left(x_{i}-\bar{x}\right)^{2}}{n-1}}$

ii) The range $w=x_{\text {nax }}-x_{\min }$

iii) The mean deviation $d=\frac{1}{n} \Sigma\left|x_{i}-\bar{x}\right|$, and apply the two procedures to compare the relative merits of $s$ and $w$ and of $s$ and $d$. According to $H_{l}$, the right tails of the distributions of these estimators will be used as critical regions, $i_{\circ} e$. we will reject $H_{0}$ when $s>s_{\alpha}, w>w_{\alpha}$ or $d>d_{\alpha}$ for an a level of significance.

Since $\frac{(n-1) s^{2}}{\sigma^{2}}$ is distributed as a $\chi^{2}$ with $n-1$ degrees of freedom, the percentage points of $s^{2}$, and hence of $s$, can be obtained from those of the $\chi^{2}$ distribution. The percentage points of $w$ and $d$ are given in Biometrika Tables (tables 22,21).

The probability of obtaining significance with w at level $\beta$, given that $s$ is just significant at level $\alpha$, is

$$
\begin{aligned}
P_{W} & =\operatorname{Pr}\left(w>w_{\beta} \mid s=s_{\alpha}\right) \\
& =\operatorname{Pr}\left(\frac{w}{s}>\frac{w_{G}}{s} \mid s=s_{\alpha}\right) \\
& =\operatorname{Pr}\left(\frac{w}{s}>\frac{w_{B}}{s_{\alpha}}\right)
\end{aligned}
$$


This last step is justified by the independence of $\frac{W}{S}$ and $s$ which can be proved as follows:

By applying Helmert's orthogonal transfornation to the $x$ 's of our sample we showed that

$$
y_{1}=\bar{x} \vee n
$$

and

$$
\Sigma\left(x_{i}-\bar{x}\right)^{2}=\sum_{j=2}^{n} y_{j}^{2}
$$

$y_{1}$ being independent of $y_{2}, y_{3}, \ldots, y_{n}$.

Since $\quad \Sigma\left(x_{i}-\bar{x}\right)^{2}=(n-1) s^{2}$

hence $\quad \Sigma y_{j}^{2}=(n-I) s^{2}$

and $x_{i}-\bar{x}$ is a function of the $y_{j}$

The range w can be written as

$$
\begin{aligned}
w & =\left(x_{\max }-\bar{x}\right)-\left(x_{\min }-\bar{x}\right) \\
& =\max \left(x_{i}-\bar{x}\right)-\min \left(x_{i}-\bar{x}\right)
\end{aligned}
$$

which is also a function of the $y_{j}$ and hence independent of $\mathrm{y}_{I}=\overline{\mathrm{x}} \mathrm{V} \mathrm{n}$.

We now make the polar transformation

$$
\begin{aligned}
& y_{2}=R \cos \theta_{1} \cos \theta_{2} \cdots \cdots \cdot \cos \theta_{n-2} \\
& y_{3}=R \cos \theta_{1} \cos \theta_{2} \cdots \cdot \cos \theta_{n-3} \sin \theta_{n-2} \\
& y_{1}=R \cos \theta_{1} \cos \theta_{2} \cdots \cdot \sin \theta_{n-3} \\
& \vdots \\
& y_{n}=R \sin \theta_{1} .
\end{aligned}
$$


Therefore

and

$$
\sum_{i=2}^{n} y_{i}^{2}=(n-1) s^{2}=R^{2}
$$

$$
s=\frac{R}{\sqrt{ }(n-1)}
$$

Since

$$
\begin{aligned}
\frac{w}{s} & =\frac{f\left(y_{2}, \ldots, y_{n}\right)}{s}=\frac{R f_{1}\left(\theta_{1}, \theta_{2}, \ldots, \theta_{n-2}\right)}{\frac{R}{V(n-1)}} \\
& =V(n-1) f\left(\theta_{1}, \theta_{2}, \ldots, \theta_{n-2}\right)
\end{aligned}
$$

then $\frac{W}{S}$ is independent of $R$ and of $s$.

In order to evaluate (2) it is necessary to have some knowledge of the distribution of $\frac{W}{S}$. This distribution can be approximated by a Pearson type irequency curve if its first four rnoments are set equal to the first four moments of a particular Pearson-type curve.

To determine what type of Pearson distribution is appropriate we use the chart given as Table 43 (Biometrika Tables). In it, the "moment coefficients", $\beta_{1}=\frac{\mu_{3}^{2}}{\mu_{2}^{2}}$, $B_{2}=\frac{\mu_{1}}{\mu_{2}^{2}}$, are plotted on rectangular axes and the seven main types of Pearson curves can be associated with points, lines, or areas in that plane. Hence, our problem is to find $\beta_{1}\left(\frac{w}{S}\right)$ and $\beta_{2}\left(\frac{W}{S}\right)$.

The moments of $\frac{W}{5}$ about the origin can be obtained from the relation 


$$
\mu_{r}^{r}\left(\frac{W}{S}\right)=\mathscr{C}\left(\frac{W}{S}\right)^{r}=S\left(\frac{W^{r}}{S^{r}}\right)=\frac{\mathscr{C}\left(W^{r}\right)}{\mathscr{C}\left(s^{r}\right)}=\frac{\mu_{r}^{r}(w)}{\mu_{r}^{r}(s)} .
$$

This can be justified by noting that

$$
\mathscr{U}\left(w^{r}\right)=\mathcal{E}\left(\frac{w^{r}}{s^{r}} \cdot s^{r}\right)=\mathscr{C}\left(\frac{w^{r}}{s^{r}}\right) \cdot \mathscr{C}^{B}\left(s^{r}\right)
$$

which follows from the independence of $\frac{\mathrm{W}}{\mathrm{s}}$ and $\mathrm{s}$.

The moments $\mu_{r}(s)$ are calculated directly from the frequency distribution of $s$, which can be derived from the $\chi^{2}$ function. The moments $\mu_{r}(w)$ are determined from the moments about the mean $\mu_{r}(w)$ by aplying familiar relationships. After (3) has been evaluated the reverse relationships aro used to obtain $\mu_{r}\left(\frac{W}{S}\right)$ from $\mu_{r}\left(\frac{W}{S}\right)$.

For $n=10, \beta_{1}\left(\frac{w}{S}\right)$ and $\beta_{2}\left(\frac{w}{S}\right)$ were found to be .013 and 2.62 respectively, determining a point which falls in the Pearson Type I area of the chart mentioned above. ( $)_{\text {) Since }}$ the probability integral of this curve can be obtained from the probability integral of the incomplete $B$ - function by making a simple change of variables, it is possible to evaluate $P_{W^{\circ}}$

$$
\begin{aligned}
\text { When } n=10, a=0.01, \beta=0.05, \text { we have } \\
P_{W}=\operatorname{Pr}\left(\frac{W}{S}>2.83 I\right)=.809 .
\end{aligned}
$$

(*) The calculation of the constants of the Pearson type I distribution was made from formulas given by W.P. Elderton (1938). 
The distribution of $\frac{W}{S}$ has been shown to be of a bounded nature (Thomson) and for $n=3, \frac{\mathrm{w}_{\beta}}{\mathrm{s}_{\alpha}}$ is less than the lower bound of $\frac{\mathrm{W}}{\mathrm{s}}$ which is equal to $/ 3$. Therefore in this case $\mathrm{P}_{\mathrm{W}}$ is unity.

$P_{d}$, the probability of establishing significance with the mean deviation at a level $\beta$ when $s$ is just significant at level $a$, can be determined by the same approach as $\mathrm{P}_{\mathrm{W}}{ }^{\circ}$ Thus, for $n=10, a=0.01, \beta=0.05$

$$
\begin{aligned}
P_{d} & =\operatorname{Pr}\left(\frac{d}{s}>\frac{d}{s_{d}}\right) \\
& =\operatorname{Pr}\left(\frac{d}{s}>0.6997\right) \\
& =0.905 .
\end{aligned}
$$

As in the case of $\frac{W}{S}$, the distribution of $\frac{d}{s}$ is approximated by a Pearson Type $I$ curve, and $P_{d}$ is evaluated from the probability integral of the Incomplete B-function.

For $n=3$, the bounds of the distribution $\frac{d}{s}$ can be found by maximizing - or minimizing - the ratio

$$
\frac{d}{s}=\frac{\frac{1}{3} \sum_{i=1}^{3}\left|x_{i}-\bar{x}\right|}{\sqrt{\frac{\sum_{i=1}^{3}\left(x_{i}-\bar{x}\right)^{2}}{2}}} .
$$

Since the maximum (or minimum) does not depend on the origin and scale of the $x_{1}$, we may assume $x_{1}=0$ and $x_{3}=1$. We may also assume, without loss of generality, that $x_{2} \geq \frac{1}{2}$ which 
enables us to write d as follows:

$$
\begin{aligned}
d & =\frac{1}{3}\left\{10-\frac{x_{2}+1}{3}|+| x_{2}-\frac{x_{2}+1}{3}|+| 1-\frac{x_{2}+1}{3} \mid\right\} \\
& =\frac{2}{9}\left(x_{2}+1\right) .
\end{aligned}
$$

Hence

$$
\frac{d}{s}=\frac{2 \sqrt{3}\left(x_{2}+1\right)}{9 \sqrt{x_{2}^{2}-x_{2}+1}}
$$

Taking the derivative of $\frac{d}{s}$ with respect to $x_{2}$ and setting it equal to zero, a maximum is found for $x_{2}=1$. The upper bound of $\frac{d}{s}$ is then $\frac{4}{3 \sqrt{ } 3}$. Since the function is increasing, a minimum is obtained when $x_{2}=\frac{1}{2}$, the lower bound being then equal to $\frac{2}{3}$. In this case $\frac{d_{\beta}}{s_{\alpha}}=0.595$ and therefore $P_{d}$ is also 1 .

The "equivalent Type I error" of the range, $\gamma_{w}$, can be determined by noting that

$$
\begin{aligned}
E\left(w / s=s_{\alpha}\right) & =\mathscr{E}\left(\frac{w}{s} \cdot s \mid s=s_{\alpha}\right) \\
& =s_{\alpha} E\left(\frac{w}{s} \mid s=s_{\alpha}\right) \\
& =s_{C} \mathbb{E}\left(\frac{w}{s}\right) \\
& =s_{\alpha} \frac{E(w)}{\mathcal{E}(s)},
\end{aligned}
$$

the third aind fourth steps being justified by the independence of $\frac{\mathrm{y}}{\mathrm{s}}$ and $\mathrm{s}$.

Hence

$$
r_{\mathrm{W}}=\operatorname{Pr}\left[\mathrm{w}>\mathrm{s}_{\alpha} \frac{\tilde{g}(\mathrm{w})}{\mathrm{g}(\mathrm{s})}\right] .
$$


Similarly, we obtain

$$
r_{d}=\operatorname{Pr}\left[d>s_{\alpha} \frac{\mathscr{g}(d)}{E(s)}\right]
$$

The probability integral of the range is given in

Table 23 (Biometrika Tables) and tables of the probability integral of the mean deviation are given by Godwin and Hartley (Biometrika, 1945). The values of $\mathcal{C}(w), \mathcal{C}(d)$ are taken from Table 20 and $\mathfrak{E}(s)$ from Table 35 of Biometrika Tables.

Table 3. below gives $\gamma_{w}$ and $r_{d}$ for $a=0.05,0.01$ and $n=3(1) 10$. The values of $\gamma_{d}$ for $n=\infty$ were obtained from the formula $\gamma=\operatorname{Pr}\left(u>\rho u_{\alpha}\right)$ remembering that the mean deviation tends to normality as $n$ becomes larger. The correlation between $s$ and $d$ is

$$
\rho=\sqrt{ } E_{d},
$$

where $E_{d}$ is the relative efficiency of the two estimators.

Formula (4) can be proved as follows:

$$
\rho(s, d)=\frac{E(s d)-E(s) E(d)}{\sigma(s) \sigma(d)} .
$$

Now, since $\frac{d}{s}$ is independent of $s$,

$$
\begin{aligned}
\mathfrak{C}(s d) & =\mathcal{C}\left(s^{2} \cdot \frac{d}{s}\right)=\mathcal{C}\left(s^{2}\right) \cdot \mathcal{E}\left(\frac{d}{s}\right) \\
& =\mathcal{E}\left(s^{2}\right) \cdot \frac{\mathcal{E}(d)}{\mathcal{E}(s)} .
\end{aligned}
$$

$\overline{(*) \text { Compare H. O. Hartley (1955) }}$ 
Therefore

$$
\begin{aligned}
\rho(s, d) & =\frac{\mathcal{E}^{\delta}\left(s^{2}\right) \frac{\mathcal{E}(d)}{\mathcal{E}(s)}-\mathcal{E}(s) \mathcal{E}(d)}{\sigma(s) \sigma(d)} \\
& =\frac{\frac{\mathcal{E}(d)}{\varepsilon(s)}\left\{\tilde{E}\left(s^{2}\right)-[\mathcal{E}(s)]^{2}\right\}}{\sigma(s) \sigma(d)} \\
& =\frac{\varepsilon(d)}{\varepsilon(s)} \cdot \frac{\sigma^{2}(s)}{\sigma(s) \sigma(d)} \\
& =\frac{\mathscr{E}(d)}{\mathcal{E}(s)} \cdot \frac{\sigma(s)}{\sigma(d)} \\
& =\sqrt{E} .
\end{aligned}
$$

Table 3. Values of the "equivalent Type I errors"

$r_{w}, r_{d}$, of tests based on the range $w$ and the mean

deviation d corresponding to Type I error a in standard $\left(x^{2}\right)$ test

\begin{tabular}{|c||c|c||c|c|}
\hline n & $\begin{array}{c}0.05 \\
r_{\mathrm{w}}\end{array}$ & $\begin{array}{c}0.01 \\
r_{\mathrm{w}}\end{array}$ & $\begin{array}{c}0.05 \\
r_{\mathrm{d}}\end{array}$ & $\begin{array}{c}0.01 \\
r_{\mathrm{d}}\end{array}$ \\
\hline 3 & 0.0508 & 0.0105 & 0.0508 & 0.0105 \\
4 & .0526 & .0114 & .0536 & .0122 \\
5 & .0545 & .0125 & .0557 & .0130 \\
6 & .0571 & .0136 & .0569 & .0136 \\
7 & .0594 & .0149 & .05785 & .0139 \\
8 & .0617 & .0161 & .0535 & .0141 \\
9 & .0641 & .0174 & .0589 & .0143 \\
10 & .0664 & .0186 & .0593 & .0145 \\
$\infty$ & - & - & .0618 & .0147 \\
\hline
\end{tabular}


IV. THE SIGN TEST AND THE T-TEST

If we have a randon sample of in observations from a normal population with $\sigma^{2}$ unknown and want to test $H_{0}: \mu=0$ against $H_{1}: \mu>0$, the use of the statistic

$$
i=\frac{\bar{x} V_{\text {in }}}{s}
$$

provides a uiformly most powerful test for that mill hypothesis. The critical region in this case is

$$
t>t_{\alpha}
$$

where $t_{\alpha}$ is the upper a significance point of a t-distribution with $n-1$ degrees of freedom.

The sign test is useful when the assumptions required for the t-test cannot be made (e.g. assumption of normality), and because of its simplicity is sometimes preferred even when the t-test is applicable. To apply the sign test to the above situation, we count the number, $r$, of positive observations. If $H_{0}$ is true, $r^{\prime}$ is a binomial variate with $p=\frac{3}{2}$ 。 We now apply our first method to a comparison of the sign test and the t-test.

The probability that $x_{i}>0$, given that the t-test is just significant at level $\alpha$, is

$$
\begin{aligned}
\pi_{c} & =\operatorname{Pr}\left(x_{i}>0 \mid t=t_{\alpha}\right) \\
& =\operatorname{Pr}\left(\frac{x_{i}}{s}>0 \mid \frac{\bar{x} \sqrt{ } n}{s}=t_{a}\right)
\end{aligned}
$$




$$
=\operatorname{Pr}\left(\frac{x_{i}-\bar{x}}{s}+\frac{\bar{x}}{s i}>0 \mid \frac{\bar{x} \vee n}{s}=t_{\alpha}\right) .
$$

Since $\frac{x_{i}-\bar{x}}{j}$ is independent of $\bar{x}$ and $s$ and therefore independent of \pm (a function of $\bar{x}, s)$ it follows that

$$
\begin{aligned}
\pi_{c} & =\operatorname{Pr}\left(\frac{x_{i}-\bar{x}}{3}+\frac{t_{\alpha}}{\sqrt{n}}>0\right) \\
& =\operatorname{Pr}\left(\frac{x_{i}-\bar{x}}{s}>-\frac{t_{Q}}{\sqrt{n}}\right) .
\end{aligned}
$$

However, the probability of $\underline{\underline{r}}$ "successes" out of a trials is difficult to evaluate because the $n$ trials are not independent. But the conditional probability of $n$ straight successes is

$$
P^{\prime}=\operatorname{Pr}\left(\frac{x_{\min }-\bar{x}}{s}>-\frac{t_{a}}{\sqrt{n}}\right)=\operatorname{Pr}\left(\frac{x_{\max }-\bar{x}}{s}<\frac{t_{\alpha}}{V_{n}}\right) .
$$

$\mathrm{P}^{\prime}$ can be evaluated by intesrating the probability density funcition of $\frac{x_{\max }-\bar{x}}{s}$ wich has been determined by Borenius (1958).

The runction $\frac{x_{\text {max }}-\bar{x}}{s}=r$ is bounded and for sample sizes $4,5,6$ takes the following forms: $\mathrm{n}=4$

$$
\begin{aligned}
f(r) & =\frac{2}{3} & 1 \leq r \leq \sqrt{ } 3 \\
& =\frac{2}{\sqrt{3}}\left[1-\frac{3}{\pi} \tan ^{-1} \frac{\sqrt{3\left(1-r^{2}\right)}}{r \sqrt{ } 2}\right] & \frac{1}{\sqrt{3}} \leq r \leq 1
\end{aligned}
$$


$\mathrm{n}=5$

$$
\begin{aligned}
f(r) & =\frac{5}{2 \pi}\left\{2 r \sqrt{\frac{5}{3}}-\sqrt{4-r^{2}}\right\} & \sqrt{\frac{2}{3}} \leq r \leq \sqrt{\frac{3}{2}} \\
& =\frac{5}{2 \pi} \sqrt{4-r^{2}} & \sqrt{\frac{3}{2}} \leq r \leq 2
\end{aligned}
$$

$n=6$

$$
\begin{array}{rlrl}
f(r) & =\frac{9}{10 \sqrt{ } 5}\left(5-r^{2}\right) & \sqrt{ } 2 \leq r \leq \sqrt{ } 5 \\
& =\frac{9}{10 \sqrt{ } 5}\left(5-r^{2}\right)+\frac{9 \sqrt{ } 3}{4 \pi} r \sqrt{2-r^{2}} & \\
& -\frac{9}{2 \pi \sqrt{ } 5}\left(5-r^{2}\right) \tan ^{-1} \frac{\sqrt{5\left(2-r^{2}\right)}}{r \sqrt{ } 3} \quad 1 \leq r \leq \sqrt{ } 2 \\
& =\frac{9}{10 \sqrt{ } 5}\left(5-r^{2}\right)+\frac{9 \sqrt{ } 3}{2 \pi}\left\{\frac{\left.r^{2}-\frac{r}{2} \sqrt{2-r^{2}}\right\}}{2 \pi \sqrt{ }}\left(5-r^{2}\right)\left\{\tan ^{-1} \quad \frac{\sqrt{5\left(2-r^{2}\right)}}{r \sqrt{ } 3}-2 \tan ^{-1} \sqrt{\frac{5}{3}}\right\}\right. \\
& +\frac{9}{\sqrt{2}} \leq r \leq 1
\end{array}
$$

Nost of these integrals are readily evaluated by standard procedures.

The integral of $\tan ^{-1} \frac{\sqrt{5\left(2-r^{2}\right)}}{r \sqrt{ } 3}$ can be evaluated by letting $\frac{\sqrt{5\left(2-r^{2}\right)}}{r \sqrt{ } 3}=t$ and integrating by parts which reduces it to the form

$k \int \frac{d t}{\left(1+t^{2}\right) \sqrt{5+3 t^{2}}}$. 
This can then be integrated by performing a second transformation, viz. $1+t^{2}=\frac{1}{w}$ to give

$$
-\frac{1}{2 \sqrt{ } 2} \sin ^{-1}\left(\frac{12 w+1}{5}\right) \text {. }
$$

The final result is then

$$
\sqrt{ } 10\left[\frac{\tan ^{-1} t}{\sqrt{5+3 t^{2}}}+\frac{1}{2 \sqrt{ } 2} \sin ^{-1}\left(\frac{t^{2}+5}{5\left(t^{2}+1\right)}\right)\right] \text {. }
$$

By the same procedure

$$
\int r^{2} \tan ^{-1} \frac{\sqrt{5\left(2-r^{2}\right)}}{r \sqrt{3}} d r
$$

can be evaluated, but in this case a third transformation is needed. Thus, after integration by parts we are left with an integral of the form

$$
k \int \frac{d t}{\left(1+t^{2}\right)\left(5+3 t^{2}\right)^{3 / 2}}
$$

which, by letting $I+t^{2}=\frac{I}{w}$, is transformed into

$$
k^{\prime} \int \frac{w d w}{\sqrt{1-w}(2 w+3)^{3 / 2}} \text {. }
$$


Our last step is to let $2 \mathrm{w}+3=\mathrm{y}^{2}$. Equation (6) is then equal to

$$
k^{\prime \prime}\left[\sin ^{-1} \frac{y}{\sqrt{5}}+\frac{3 \sqrt{5-y^{2}}}{5 y}\right]
$$

and the final result is

$$
\begin{aligned}
10 \sqrt{ } 10 & \left\{\frac{\tan ^{-1}}{3\left(5+3 t^{2}\right)^{3 / 2}}+\frac{1}{6 \sqrt{ } 2} \sin ^{-1} / \frac{5+3 t^{2}}{5\left(1+t^{2}\right)}\right. \\
& \left.+\frac{1}{10 \sqrt{ } 2} \sqrt{5+3 t^{2}}\right\}
\end{aligned}
$$

where $t=\frac{\sqrt{5\left(2-r^{2}\right)}}{r \sqrt{ } 3}$ as before.

Table 4 gives $P^{\prime}$ for $\alpha=.05, .01$ and $n=4,5,6$. In the case $n=4, \alpha=.01$, the value of $\frac{\alpha}{\sqrt{ } n}$ falls beyond the

\begin{tabular}{|c|c|c|c|}
\hline $\mathrm{n}$ & $\alpha=0.05^{P}$ & $c=0.01$ & $\begin{array}{l}\text { Significance level } \\
\text { of sign test }\end{array}$ \\
\hline 4 & 0.359 & 1 & $1 / 16$ \\
\hline 5 & 0.074 & 0.809 & $1 / 32$ \\
\hline 6 & 0.055 & 0.417 & $1 / 64$ \\
\hline
\end{tabular}
upper bound, $\sqrt{ } 3$, of the function and therefore $p^{\prime}$ equals unity.

TABLE 4 
Using the second approach, we will now determine the "equivalent Type I error" of the sign test. From (5), the expected number of positive $x^{\prime} s$ is

$$
\begin{aligned}
g(r) & =n \pi_{c} \\
& =n \operatorname{Pr}\left(-\frac{x_{1}-\bar{x}}{s}>-\frac{t_{\alpha}}{\sqrt{ } n}\right),
\end{aligned}
$$

where $t_{\alpha}$ is based on $n-I$ degrees of freedom. Let $y=\frac{x_{1}-\bar{x}}{s}$; then

$$
y^{2}=\frac{\left(x_{1}-\bar{x}\right)^{2}}{\frac{I}{n-1} \sum\left(x_{i}-\bar{x}\right)^{2}} .
$$

But

$$
\sum_{i=1}^{n}\left(x_{i}-\bar{x}\right)^{2}=\sum_{j=2}^{n}\left(x_{j}-\bar{x}_{n-1}\right)^{2}+\frac{n}{n-I}\left(x_{1}-\bar{x}\right)^{2}
$$

where $\quad \bar{x}_{n-1}=\frac{\sum_{j=2}^{n} x_{j}}{n-1}$.

The first term on the right hand side is distributed as $\chi_{n-2}^{2} \sigma^{2}$ and the second as $\chi_{1}^{2} \sigma^{2}, i . e .$, is the square of a unit normal variable. Since the left hand term is distributed as $x_{n-1}^{2} \sigma^{2}$, it follows that the two terms on the right are statistically independent. We have, therefore,

$$
y^{2}=\frac{(n-1)\left(x_{1}-\bar{x}\right)^{2}}{x_{n-2}^{2} \sigma^{2}+\frac{n}{n-1}\left(x_{1}-\bar{x}\right)^{2}}
$$




$$
\begin{aligned}
& =\frac{\frac{(n-1)^{2}}{n} \cdot \frac{\frac{n}{n-1}\left(x_{1}-\bar{x}\right)^{2}}{\frac{x_{n-2}^{2} \sigma^{2}}{n-2}}}{(n-2)+\frac{\frac{n}{n-1}\left(x_{1}-\bar{x}\right)^{2}}{\frac{x_{n-2}^{2}}{n-2}}} \\
& =\frac{\frac{(n-1)^{2}}{n} t_{n-2}^{2}}{(n-2)+t_{n-2}^{2}}=\frac{(n-1)^{2} t_{n-2}^{2}}{n\left(n-2+t_{n-2}^{2}\right)} .
\end{aligned}
$$

Let $c=\frac{t_{\alpha}}{\sqrt{n}}$. Then

$$
\begin{aligned}
\pi_{c} & =\operatorname{Pr}(y>-c) \\
& =\operatorname{Pr}(y>0)+\operatorname{Pr}(-c<y<0) \\
& =\frac{3}{2}+\frac{1}{2} \operatorname{Pr}\left(y^{2}<c^{2}\right) .
\end{aligned}
$$

From (7) we have

$$
\frac{n(n-2) y^{2}}{(n-1)^{2}-n y^{2}}=t_{n-2}^{2}
$$

and since this is a monotone increasing function of $y^{2}$, we can write

$$
\begin{aligned}
\operatorname{Pr}\left(y^{2}<c^{2}\right) & =\operatorname{Pr}\left(\frac{n(n-2) y^{2}}{(n-1)^{2}-n y^{2}}<\frac{n(n-2) c^{2}}{(n-2)^{2}-n c^{2}}\right) \\
& =\operatorname{Pr}\left(t_{n-2}^{2}<k^{2}\right) \\
& =2 \operatorname{Pr}\left(0<t_{n-2}<k\right),
\end{aligned}
$$

provided $(n-1)^{2}-n y^{2}>0$

$$
(n-1)^{2}-n c^{2}>0 \text {. }
$$


Hence

$$
\begin{aligned}
\pi_{c} & =\frac{3}{2}+\operatorname{Pr}\left(0<t_{n-2}<k\right) \\
& =\operatorname{Pr}\left(t_{n-2}<k\right) \\
& =\operatorname{Pr}\left(t_{n-2}<\sqrt{\frac{(n-2) t_{\alpha}^{2}}{(n-1)^{2}-t_{\alpha}^{2}}}\right) \\
& =\operatorname{Pr}\left(t_{n-2}<t_{\alpha} \sqrt{\frac{n-2}{(n-1)^{2}-t_{\alpha}^{2}}}\right) .
\end{aligned}
$$

The minimum of $\frac{x_{1}-\bar{x}}{s}$ is $-\left(\frac{n-1}{\sqrt{ } n}\right)$ so when $n-1<t_{\alpha}$ we get $\pi_{c}=1$

We can now evaluate $r=\operatorname{Pr}\left(r>n \pi_{c}\right)$ by making the histogram representation of the binomial distribution of $r$ (parameters $n$, 新). This representation is necessary since $\mathrm{n} \pi_{\mathrm{c}}$ is, in general, not an integer. If we denote by $I$ the integral part of $n \pi_{c}+\frac{1}{2}$,

$$
r=\operatorname{Pr}(r \geq I+I)+\left(n \pi_{c}+\frac{3}{2}-I\right) \operatorname{Pr}(r=I) \text {. }
$$

For large values of $\mathrm{n}$ the normal approximation to the binomial is quite satisfactory. Hence

$$
\gamma \stackrel{\dot{b}}{*} \operatorname{Pr}\left[u>\sqrt{ } n\left(2 \pi_{c}-1\right)\right] \text { for large } n \text {. }
$$

In Table $5 \gamma$ is given for several values of $\gamma=n-2$ and $a$. Some irregularities in the values obtained can be attributed to the discreteness of the variable $\underline{r}$. 
Table 5. Equivalent Type I error $x$ of the sign test corresponding to the paired t-test in samples of $n$ with Type I $\underline{\operatorname{error} \alpha}$

\begin{tabular}{|c|c|c|c|c|}
\hline$v=n-2$ & 0.05 & 0.025 & 0.01 & 0.005 \\
\hline 2 & 0.0582 & 0.0312 & 0.0312 & 0.0312 \\
\hline 3 & .0918 & .0308 & .0171 & .0156 \\
\hline 4 & .0892 & .0522 & .0145 & .0103 \\
\hline 5 & .0912 & .0501 & .0240 & .0077 \\
\hline 6 & .0971 & .0518 & .0245 & .0142 \\
\hline 7 & .0372 & .0570 & .0211 & .0138 \\
\hline 8 & .0972 & .0511 & .0278 & .0119 \\
\hline 9 & .0945 & .0574 & .0262 & .0161 \\
\hline 10 & .0939 & .0568 & .0264 & .0152 \\
\hline 12 & .0596 & .0587 & .0263 & .0171 \\
\hline 15 & .0956 & .0577 & .0271 & .0174 \\
\hline 20 & .0964 & .0576 & .0281 & .0182 \\
\hline 30 & .0950 & .0565 & .0301 & .0187 \\
\hline 60 & .0950 & .0588 & .0308 & .0193 \\
\hline$\infty$ & .0947 & .0589 & .0317 & .0199 \\
\hline
\end{tabular}




\section{ACKNOWLEDGNEINTS}

The author wishes to express her sincere appreciation to for his guidance and advice in preparing this thesis. His invaluable assistance made this paper possible.

The author wishes also to thank for his helpful criticism and suggestions. Thanks are also extended to who prepared the final typewritten copies. 


\section{RISERENCES}

Borenius, G. (1950). On the distribution of the extreme values in a sample from a normal distribution. skand. Aktuarietedskx. 131-66.

David, H. A., Perez, C. A. (1959). On comparing tests of the same hypothesis. Technical Report No. 44. Dept. of Statistics and Statistical Laboratory. Virginia Polytechinic Institute.

David, A. A., Hartley, H. O., and Pearson, E. S. (1954). The distribution of the ratio, in a single normal sample, of range to standard deviation. Biometrika, 41, 482-93. Elderton, W. P. (1938). Frequency Curves and Correlation. Cambridge University Press, p. 57-58. Godwin, H. Jo and Hartley, H. O. (1945). Probability integral and percentage points of the mean deviation in normal samples. Biometrika, 33, 254-65. Hartley, H. O. (1955). Some recent developments in analysis of variance. Comm. Pure and Appl. Math. $8,47-72$. Thomson, G. W. (1955). Bounds for the ratio of range to standard deviation. Biometrika, 42, 268-269. 


\section{The vita has been removed from the scanned document}




\section{ABSTRACT}

This thesis presents two alternative procedures for comparing standard and quick tests of a null hypothesis $\mathrm{H}_{0}$ • This comparison is usually made by plotting the power curves of each test for a fixed Type I error. However, the power curves give only an indication of the individual performances of each test and not of the extent to which they agree when applied to the same problem. The procedures discussed in this paper deal with determining this degree of agreement. The first method determines the probability, $P$, that the quick test leads to a significant result at a level $\ddot{\beta}$, given that the standard test jo just significant at level $\alpha$. If the standard and quick tests are based on the statistics $u_{1}$ and $u_{2}$, respectively, the second approach determines the level of significance corresponding to the expected value of $u_{2}$ given that $u_{1}$ is just significant at level $\alpha$. This level of significance is termed the "equivalent Type I error" of the quick test and denoted by $\gamma$.

Both methods are applied to compare tests of location, dispersion, and the paired t-test with the sign test, all in samples taken from a normal population. In the first two cases, values of $P$ and $\gamma$ are given for different sample sizes, and in the third case only the "equivalent Type I error" of the sign test is given, $P$ being rather difficult to evaluate. 